\title{
Responder Sin Exclusiones a las Necesidades Educativas de las Altas Capacidades
}

\section{Answer Without Exclusion to the Educational Needs of Gifted Students}

Elena Hernández de la Torre *, Ma José Navarro Montaño

Universidad de Sevilla, España

\section{DESCRIPTORES:}

Educación en derechos humanos

Capacidad intelectual Educación para todos Estrategias de enseñanza Práctica de enseñanza Rendimiento académico

\section{RESUMEN:}

El objetivo de este estudio es indagar en las respuestas educativas ofrecidas por el profesorado y los propios centros a los requerimientos escolares de este alumnado con altas capacidades. En esta investigación han participado los propios protagonistas, profesorado y alumnado, ya que su opinión se considera imprescindible para conocer la atención que actualmente se les ofrece y si responde a las necesidades que se presentan cotidianamente en los centros educativos respecto a este alumnado. Hemos utilizado una metodología enfoque cualitativo con entrevistas de opinión a docentes de centros de prácticas Educación Primaria y Secundaria, así como a estudiantes de Secundaria y Bachillerato con AACC que han aceptado participar. Los resultados destacan la necesidad de trabajar los aspectos emocionales y habilidades sociales con este alumnado para su inclusión educativa y evitar la segregación, el diseño de respuestas inclusivas, no separadas y ajustadas a sus exigencias a través de Programas de Enriquecimiento y/o flexibilidad del currículum de forma abierta y flexible, la necesidad asimismo de coordinación entre profesionales para trabajar y diseñar respuestas innovadoras, nuevas estrategias de enseñanza en el aula y recursos educativos para trabajar mejor, formación específica para el profesorado en esta atención educativa y atención al contexto familiar.

\section{KEYWORDS:}

\section{ABSTRACT:}

Intellectual capacity Education for all Teaching strategies Teaching practice Student performance
The objective of this study is to investigate the educational responses offered by teachers and the schools themselves to the school requirements of this student with high capacities. In this research the protagonists themselves, teachers and students have participated, since their opinion is considered essential to know the attention that is currently offered to them and if it responds to the needs that are presented daily in the educational centers regarding this student body. We have used a qualitative approach methodology with opinion interviews with teachers of Primary and Secondary Education practice centers as well as Secondary and Baccalaureate students with AACC who have agreed to participate. The results highlight the need to work on emotional aspects and social skills with this student for their educational inclusion and avoid segregation, the design of an inclusive and non-segregating responses tailored to their requirements through enrichment programs and / or flexibility of the curriculum in an open and flexible way, the need for coordination among professionals to work better and design innovative responses, new teaching strategies in the classroom and educational resources to work better, specific training for teachers in this educational care and attention to context family.

\section{CÓMO CITAR:}

Hernández de la Torre, E. y Navarro Montaño, M. J. (2021). Responder sin exclusiones a las necesidades educativas de las altas capacidades. REICE. Revista Iberoamericana sobre Calidad, Eficacia y Cambio en Educación, 19(3), 5-18. https://doi.org/10.15366/reice2021.19.3.001

*Contacto: eht@us.es

ISSN: 1696-4713

revistas.uam.es/reice 


\section{Introducción}

Este artículo tiene como objetivo indagar en las respuestas de docentes y alumnado de Altas Capacidades (en adelante AACC) respecto a la atención educativa ofrecida en los centros educativos, siendo un tema de preocupación en la comunidad educativa. Las AACC se atienden actualmente según propuestas particulares de profesorado y centros debido a la carencia de actuaciones satisfactorias por razones relacionadas con la formación del profesorado, recursos personales, materiales y estructurales, insuficiente tiempo dedicado a este alumnado, estrategias de abordaje y un sistema educativo que es excesivamente lineal, poco flexible y demasiado estricto.

El hecho de que "no exista una teoría determinante que permita catalogar de forma inequívoca a individuos con AACC, superdotados y talentosos, ni tampoco un sistema de medida que otorgue índices de inteligencia plenamente fiables" (García-Guardia et al., 2019, p. 20) se presenta como uno de los retos a considerar. Como afirman García-Perales y Almeida (2019, p. 39), "este alumnado no es debidamente identificado, entonces también se anticipa la falta de respuestas educativas específicas para estos escolares con altas capacidades". Como consecuencia de estos motivos, profesorado y alumnado podrían encontrarse desmotivados, necesitando respuestas para mejorar esta atención educativa.

\section{Revisión de literatura}

En la actualidad la atención a este alumnado se basa en su incorporación a las aulas ordinarias para su aprendizaje y participación, aunque sin un Plan de Atención eficaz, una organización adecuada y diagnósticos concretos. Atender al alumnado con AACC supone un reto para los centros, siendo las respuestas adecuadas a sus necesidades las que se realizan mediante actividades extraescolares fuera del centro y no como actividades curriculares planificadas en el aula. Como señalan García-Guardia y otros (2019, p. 19), "durante las últimas décadas las altas capacidades (AACC) se han desvelado como un determinante curricular que evidencia la necesidad de adaptar los contenidos a las características de los alumnos definidos por las mismas”, afirmando García-Perales y Almeida (2019, p. 41) que "existe una carencia en la fundamentación de las prácticas educativas actuales con estos escolares, consideradas más un «extra» pero no una «extensión», por lo que es fundamental complementar y compactar los planes de estudio". En definitiva, "el imperativo de que la escuela debe mejorar la calidad de su propuesta no es una novedad", tal como señalan Murillo y Krichesky (2014, p. 69).

En la Ley Orgánica 8/2013 para la Mejora de la Calidad Educativa se especifica que las administraciones educativas deben adoptar medidas necesarias para identificar a este alumnado y valorar de forma temprana sus necesidades. Asimismo, deben adoptar Planes de Actuación como Programas de Enriquecimiento Curricular para que el alumnado pueda desarrollar al máximo sus capacidades. Las Instrucciones de la Dirección General de Participación y Equidad (2014) que regulan el Protocolo para la Detección y Evaluación del Alumnado con necesidades específicas de apoyo educativo por presentar AACC ofrecen información a la comunidad educativa sobre actuaciones, haciendo hincapié en las familias y debiendo cumplimentar un cuestionario para iniciar el proceso de detección.

El debate en torno a las AACC actualmente se centra en dos retos principales, la valoración del alumnado y la implementación de unos procesos de enseñanza-aprendizaje de carácter innovador. En cuanto a la valoración, Agudo (2017, p. 270) establece que "cuando un alumno/a presenta necesidades educativas asociadas a altas capacidades, lo primero es detectarlo"; se trata de pruebas que el orientador y/o psicólogo aplica directamente al niño para corroborar que el alumnado con AACC muestra un buen rendimiento cognitivo y escolar. En cuanto a los procesos de aprendizaje destaca el enriquecimiento instrumental y curricular (Higueras-Rodríguez, 2017; Torrego, 2011) por un lado y por otro ofrecer un enfoque inclusivo no segregador como respuesta a través del aprendizaje cooperativo (Torrego, Monge, Pedrajas y Martínez, 2015). El problema del desconocimiento de estos retos está generando abandono y desidia escolar basada en el estereotipo y la idea de que el alumnado con AACC dispone de habilidades cognitivas suficientes para aprender, aunque la realidad es que, en ocasiones, dejan sus estudios en ocasiones por falta de apoyo a sus necesidades. 
La respuesta centrada en el enriquecimiento curricular se concibe como "una intervención educativa que ofrece alternativas para ofrecer aprendizajes más ricos y variados a través de la modificación en la profundidad del contenido y de la metodología empleada” (Seijo, López, Pedrajas y Martínez, 2015, p. 27). Se trata de un enriquecimiento desarrollado en el contexto escolar basado en la estrategia de aprendizaje cooperativo, por lo que Fernández, De Sande y Martín (2006, p. 46) lo definen como "una amplia gama de actividades que se pueden realizar en el aula y en la organización ordinaria del centro educativo". Esta medida educativa se ubica en el aula, con diferentes metodologías y recurriendo a actividades dirigidas a todo el alumnado, a grupos reducidos o programas individuales. Además, mediante estas actividades de enriquecimiento trabajan las habilidades cognitivas y afectivas.

El enriquecimiento del programa curricular propone un "aprendizaje innovador, flexible y creativo" (Ramírez y Soto, 2012), pudiendo estar vinculado al Programa Educativo del alumno, centrarse en una o varias asignaturas y elaborando ajustes en profundidad y extensión de contenidos dentro del aula. Estos Programas de enriquecimiento "deben incluir una intervención psicológica en las áreas del desarrollo social, afectivo y emocional" (Vallejo y Morata, 2015, p. 70). En esta estrategia se encuentra el enriquecimiento extracurricular que Fernández, De Sande y Martín (2006, p. 45) definen como "programas destinados básicamente a anticipar las preguntas y los problemas de aquellos alumnos superdotados que en el contexto del aula superan el ritmo, proporcionándoles ocupaciones extra". Este enriquecimiento no responde a un enfoque inclusivo, ya que se desarrolla en contextos y horarios ajenos al ordinario (clases y/o programas específicos). Otro tipo de enriquecimiento es el enriquecimiento aleatorio, combinando contenidos curriculares y extracurriculares que tienen cierta vinculación. Martínez (2014, p. 25) establece que "es el propio alumno el que escoge los temas en función de sus intereses y motivaciones, los trabaja de forma paralela a las clases normales".

Respecto al aprendizaje cooperativo, es necesario abordarlo desde una perspectiva "normalizada e inclusiva" (Torrego et al., 2015), así "la respuesta educativa conviene realizarla en el entorno natural del alumno/a, respetando sus distintos tipos de aprendizaje y la propia realidad escolar, basada en las altas expectativas” (Palomares y García, 2016, p. 92). La atención en el aula adaptada a las necesidades y características del alumnado es fundamental para el desarrollo educativo del alumnado con AACC. No debemos olvidar que este colectivo necesita alto nivel de estimulación para desarrollar sus potencialidades eficazmente ya que "su aprendizaje puede inhibirse si no se les da la atención adecuada" (Cañete, 2010, p. 9). Delgado y Solano (2009) proponen técnicas para atender a todo el alumnado basadas en tres estrategias metodológicas: métodos de enseñanza basados en exposiciones magistrales, métodos orientados a la discusión o trabajo en equipo, juegos cooperativos, grupos interactivos, trabajos en equipo, etc. y métodos basados en aprendizaje individual.

Estas tres propuestas implican formación del profesorado para mejorar la calidad de la educación, promover el aprendizaje colaborativo y atender a la diversidad del alumnado. Su finalidad es el "desarrollo del pensamiento crítico y el debate ya que es necesario atraer al estudiante a este entorno, darle las herramientas y guías necesarias para que desarrolle las actividades y alcance exitosamente las metas propuestas" (Delgado y Solano, 2009, p.18). El análisis de estos retos se considera crucial por lo que nuestro objetivo principal en este estudio se centra en conocer las respuestas a las altas capacidades del alumnado por parte del profesorado y del alumnado para facilitar el trabajo de los profesionales.

\section{Método}

El objetivo de este estudio es indagar en las respuestas educativas del profesorado hacía el alumnado con AACC en los centros educativos. Para ello hemos utilizado una metodología cualitativa a partir de entrevistas de opinión como estrategia para responder al objetivo planteado y examinar "la forma en que los individuos perciben y experimentan los fenómenos que los rodean, profundizando en sus puntos de vista” (Hernández, Fernández y Baptista, 2014, p. 358). Pretendemos obtener de este modo opiniones de este colectivo para "comprender a las personas ya que todas las perspectivas y todos los escenarios son valiosos" (Campoy, 2016, p. 233). 
El muestreo de profesorado y alumnado ha sido no probabilístico de tipo casual; la muestra se define por disponibilidad, a propósito, y/o por conveniencia debido a que es accesible y no por criterio estadístico. Se han utilizado dos grupos de informantes: el primero se compone de 15 docentes de centros de prácticas de distintos lugares de la provincia de Sevilla de primaria y secundaria; 10 de estos centros son concertados y 5 públicos, de los cuales 12 son centros de primaria y secundaria y 3 centros de infantil y primaria. Entre el profesorado consultado 4 son profesores/as de apoyo, 2 orientadores/as y 9 profesores de primaria y secundaria.

El segundo grupo se compone de 5 estudiantes de Secundaria y Bachillerato que se ofrecen al estudio, al azar y utilizando la técnica de la bola de nieve, participando con autorización familiar y perfil homogéneo en cuanto a la edad, entre 14 y 17 años. Dos de los entrevistados cursan Educación Secundaria en centros públicos, dos Bachillerato (centro concertado/centro privado) y uno grado universitario. Las entrevistas han sido realizadas por los investigadores y han tenido una duración de 45 minutos para profesorado y para alumnado (ver Cuadro 1 del profesorado y Cuadro 2 del alumnado). Las preguntas de la entrevista al profesorado se han organizado y formulado en torno a cuatro códigos, en base a las fuentes teóricas consultadas, y que presentamos a continuación (Cuadro 1).

Para elaborar las preguntas de la entrevista del alumnado hemos utilizado las indicaciones de Guzmán y Saucedo (2015) en cuanto a los perfiles del alumnado para orientar preguntas tipo como experiencias, perfil de alumno, familia, atención escolar, profesorado y relaciones con iguales. La entrevista se ha centrado en el estudiante con AACC como sujeto de estudio teniendo en cuenta los significados como alumnado con AACC, trayectorias escolares, atención escolar recibida, identidad como estudiante, relación entre compañeros, familia, etc., siendo temáticas ampliamente estudiadas en diversas investigaciones de corte cualitativo (Guzmán y Saucedo, 2015, p.1021).

Cuadro 1

Preguntas entrevista profesorado

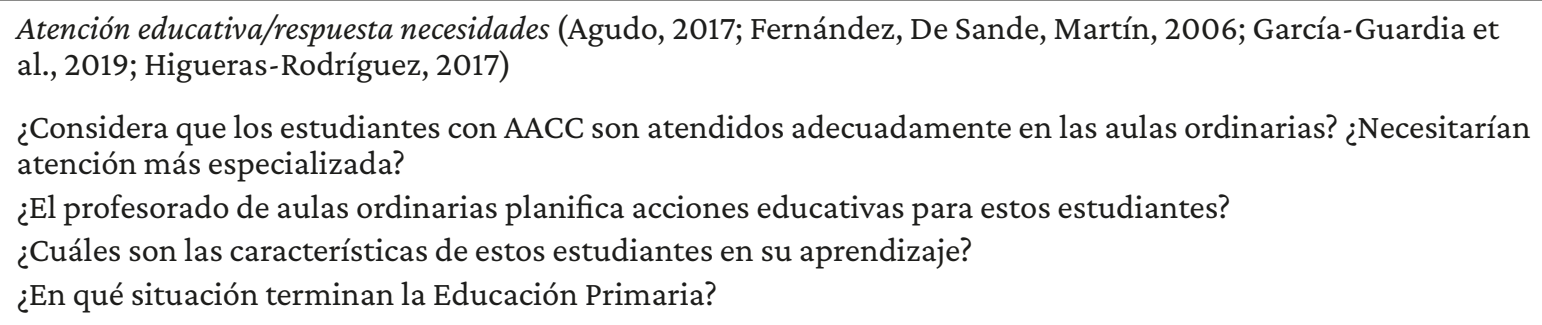

Formación profesorado sobre AACC (García-Barrera y De la Flor, 2016; Seijo et al., 2015; Torrego et al., 2015).

¿Considera que el profesorado de Educación Primaria ha recibido formación adecuada para responder a sus necesidades educativas?

¿En qué aspectos concretos cree que debería recibir formación el profesorado de Educación Primaria para responder a las necesidades de estos estudiantes?

Apoyo entre compañeros (Martínez, 2014; Palomares y García, 2016; Torrego, 2011).

¿Estos estudiantes podrían apoyar el aprendizaje de otros compañeros/as en clase? ¿Cómo son considerados por sus compañeros? ¿Comparten actividades de grupo en clase con los demás compañeros?

Relaciones familia-escuela (Castellanos et al., 2015; Higueras-Rodríguez y Fernández, 2017).

¿Conoce el profesorado el contexto familiar de estos estudiantes fuera del centro?

¿Cómo son las relaciones familia-escuela de este alumnado?

Realice propuestas para mejorar la situación educativa de estos estudiantes

Nota. Elaboración propia. 
Cuadro 2

Preguntas entrevista alumnado

¿Cómo ha sido tu experiencia durante tu vida escolar en los diferentes centros en los que has estado?

¿Cómo supiste y supieron tus padres que tenías AACC?

¿Cómo te sentiste?

¿Cómo reaccionó tu familia?

¿Se produjo algún cambio en tu vida?

¿Qué atención has recibido en el colegio y en el Instituto?

¿Ha trabajado contigo algún profesor en particular u orientador/a?

¿Te hubiera gustado trabajar en los diferentes centros de alguna otra forma?, ¿cuál?

¿Cómo han sido tus relaciones con tus compañeros de clase y fuera del centro? ¿y con los profesores?

¿Algún consejo para algún compañero a quien se detectaran AACC?

Nota. Elaboración propia.

La recogida de datos se ha realizado en distintas fases para sistematizar el trabajo a realizar en este estudio (Cuadro 3).

El análisis de los datos se ha realizado a partir de la reducción de textos en códigos basándonos en la Teoría Fundamentada, efectuando el análisis de contenido clásico y de inducción analítica y buscando una explicación más amplia a partir de las preguntas realizadas. Este análisis ha sido sistemático, a partir de una secuencia lógica y dentro de un orden (Álvarez-Gayou, 2005).

Cuadro 3

Fases de la recogida de datos

$1^{\text {a }}$ FASE: entrevistas, transcripción y elaboración de códigos y sub-códigos a partir de la información recabada (Cuadro 4/Cuadro 5). Total de entrevistas: 14 profesorado y 5 alumnado

2a FASE: categorización de información de profesorado en 4 códigos/dimensiones de análisis (Cuadro 4) y 15 códigos para alumnado (Cuadro 5) para asignar párrafos y frases o unidades de análisis (verbatim)

$3^{\text {a }}$ FASE: análisis de datos, resultados y conclusiones referidas a la atención escolar a las AACC en educación

Nota. Elaboración propia.

Los pasos que hemos seguido han sido los siguientes:

- Obtención de información: entrevistas e identificación de unidades básicas de análisis (temas)

- Transcripción y ordenamiento de la información: grabación en formato digital y papel

- Agrupación de la información en categorías/códigos para obtener ideas y opiniones con significado en párrafos organizados respecto a la temática que nos ocupa

- Composición de la información en un texto para llegar a conclusiones y elaboración de una explicación de las opiniones expresadas

Para ordenar y extraer la información se ha utilizado el Programa MXQDA 12.0 de análisis de datos cualitativos a partir de los códigos utilizados. Los códigos, así como los sub-códigos, se describen en el Cuadro 4 y Cuadro 5 referidas a las entrevistas de profesorado y alumnado. 
Cuadro 4

Códigos y Subcódigos de profesorado

\begin{tabular}{ll}
\hline \multicolumn{1}{c}{ Códigos } & \multicolumn{1}{c}{ Sub-códigos } \\
\hline & 1.1. Atención aula ordinaria (AUL/ORD) \\
& 1.2. Atención específica (ATE/ESP) \\
1-Atención educativa/respuesta & 1.3. Planificación educativa específica (PLA/ESP) \\
necesidades (ATE/RES) & 1.4. Estudiante ante el aprendizaje (EST/APRE) \\
& 1.5. Finalización de la EP (FIN/PRI) \\
& 2.1. Formación específica (FOR/ESP) \\
2-Formación del profesorado sobre AACC (FORM) & 2.2. Formación profesorado (FOR/PROF) \\
& 3.1. Ayuda entre iguales (AYU/IGU) \\
& 3.2. Inclusión en el aula (INC/AU) \\
3-Red natural de apoyo en el aula (RED) & 3.3. Trabajo colaborativo (TRA(COL) \\
& 3.4. Apoyo entre compañeros/as (APY/IGU) \\
& 4.1. Contexto familiar (CON/FAM) \\
4-Relaciones familia-escuela (FAM/ESC) & 4.2. Relaciones familia-escuela (RELFE) \\
\hline
\end{tabular}

Nota. Elaboración propia.

Cuadro 5

Códigos del análisis de entrevistas al alumnado

$\begin{array}{ll}\text { EXE } & \text { Experiencias del alumnado en su vida académica } \\ \text { EXD } & \text { Experiencia con docentes de diferentes centros educativos } \\ \text { DIAG } & \text { Procesos y pruebas de diagnóstico utilizadas } \\ \text { COM } & \text { Comunicación de diagnóstico a familias y al propio alumnado } \\ \text { FAM } & \text { Impresiones ante el diagnóstico } \\ \text { SENT } & \text { Cambios en la vida académica y social } \\ \text { CAM } & \text { Medidas de atención educativas en los centros educativos } \\ \text { MAT } & \text { Medidas de flexibilización y respuesta ante esta medida } \\ \text { FLEX } & \text { Motivación del alumnado en su vida escolar } \\ \text { MOT } & \text { Estimulación externa recibida en actividades extraescolares/familia } \\ \text { EST } & \text { Profesorado que ha atendido al alumnado de AACC de forma específica } \\ \text { PAT } & \text { Relaciones con sus iguales, dentro y fuera del centro } \\ \text { REL } & \text { Opons }\end{array}$

Nota. Elaboración propia. 


\section{Resultados}

A continuación, presentamos los resultados del análisis de entrevistas a profesorado y alumnado organizados en torno a categorías y sub-categorías.

\subsection{Análisis de las entrevistas al profesorado}

\subsubsection{Atención educativa/respuesta necesidades (ATE/RES)}

Atención aula ordinaria (AU/OR): unos aluden a que "la atención depende más de la actitud del profesorado puesto que hay maestros que no congenian ni empatizan con este colectivo" (PROF5); otros a la figura del tutor como "clave" en el proceso de enseñanza-aprendizaje (PROF9). La mayoría considera que "no están todo lo bien atendidos que deberían de estar" (PROF7), y que la atención en el aula debería mejorar (PROF11, PROF12) con la intervención de profesorado especializado (PROF13). Sin embargo, otros creen que la atención que reciben en el aula es adecuada ya que "cuentan con programas de enriquecimiento curricular" (PROF10). Otra opinión es que la respuesta educativa depende de las capacidades del alumnado, la metodología de aula (PROF14) y la utilización de recursos específicos: "trabajar por proyectos es más fácil para atenderlos" (PCA14).

Atención específica (ATE/ES): se necesitan medios personales, capacitación adecuada (PROF1) y acciones concretas: "se les aplica la flexibilización, se han adelantado un curso, pero siempre agotando otras medidas que tuviesen en cuenta los aspectos sociales y madurativos" (PROF2). Algunos centros tienen medidas específicas para mejorar esta atención en el aula, "en Primaria hay un alumno con la anticipación de un curso con buen rendimiento y realizando actividades de profundización” (PROF6). La Administración Educativa ha sido decisiva "desde que la Junta de Andalucía sacó los cuestionarios para detectarlos en $1^{\circ}$ de Primaria y en $1^{\circ}$ de Secundaria, cada vez se detectan antes"(PROF3).

Planificación educativa específica (PLA/ESP): subrayan la necesidad de ser exigentes, unificar criterios y seguir indicaciones del Dpto. de Orientación (PROF2). Algunos centros realizan adaptaciones curriculares/Programas de Enriquecimiento (PROF3) aunque no es una actuación generalizada; otros sí tienen contenidos de profundización y ampliación. En ocasiones señalan tener en cuenta la importancia de los intereses de este alumnado en la planificación educativa, "tienen fichas de ampliación/ cuadernillos específicos, dependiendo de los intereses o lo que necesiten reforzar" (PROF13), también se elaboran orientaciones recogidas en su informe psicopedagógico (PCA12), aunque "desgraciadamente son los alumnos invisibles del sistema educativo" (PROF8)

Aprendizaje del estudiante (EST/APRE): estos estudiantes no son un colectivo homogéneo, sino diverso; son estudiantes que "siempre quieren ahondar más en cada temario" (PROF1), aunque "a veces no terminan el trabajo a tiempo, se aburren y sus habilidades sociales no son del todo adecuadas, les cuesta relacionarse con sus iguales" (PROF3); a veces "no se esfuerzan en su trabajo y aprendizaje, confían demasiado en sus capacidades por lo que son considerados unos vagos por el resto de la clase" (PROF5). Sin embargo, en ocasiones se caracterizan por:

Tener vocabulario amplio, lo utilizan con exactitud, tienen mejores relaciones sociales con gente adulta, aunque les cuesta interpretar, entender y expresar sus propios sentimientos. (PROF5)

Son bastante creativos, con intereses diferentes y concretos del resto de compañeros. (PROF7)

Son muy inquietos, muy curiosos, cuando les interesa un tema se convierten en grandes expertos de ese tema, aunque tienen poca constancia. (PROF8)

Aprenden más rápido, con más facilidady de forma diferente a sus compañeros. (PROF9)

Poseen un alto nivel de energía, capacidad de concentración y persistencia, pueden ocuparse de varios temas a la vezy pueden enfocar un problema desde ópticas diferentes. (PROF9)

Razonan mejor y aprenden muy rápido, buena capacidad verbal, alto grado de dedicación a las tareas y perseverancia, realizan aprendizajes tempranos con poca ayuda. (PROF12) 
Son muy diferentes, terminan su trabajo muy rápido y bien realizado, son utilizados de compañero tutor, aunque otros son más rápidos y lo hacen mal, otros como no están dentro de sus intereses no trabajan o realizan mal la tarea mostrando dificultades. (PROF14)

En cuanto al género, "el perfil de las niñas es distinto al de los niños, son más calladas, destacan menos, son más cumplidoras... un niño con altas capacidades inadecuadamente atendido se puede convertir en un alumno disruptivo y con fracaso escolar" (PROF8).

La finalización de la EP (FIN/PRI): en Educación Primaria podrían mejorar, ya que la respuesta escolar no siempre responde a su realidad (PROF1), los resultados no se corresponden con su alto rendimiento (PROF3) aunque suelen terminar académicamente sin dificultad (PROF10) si tienen un alto nivel de motivación como consecuencia de sus buenas calificaciones (PROF9, PROF12, PROF13, PROF14). El profesorado vincula el "éxito académico con la respuesta educativa que han recibido" (PROF12). Respecto al rendimiento "es curioso porque para ser muy buenos muchos niños no destacan, se detectan en sexto de Primaria o incluso en Secundaria y eso es muy tardío" (PROF8). Las calificaciones suelen ser buenas en su mayoría, aunque el profesorado alude al fracaso escolar: "a veces puede existir fracaso escolar por la desmotivación, se aburren al escuchar cosas que ya saben o que captan a la primera y que hay que repetir para el resto de compañeros” (PROF 11).

\subsubsection{Formación del profesorado (FORM)}

Formación específica (FORM/ESP): el profesorado afirma que "tenemos nociones generales para su atención, pero nos falta formación" (PROF2, PROF3). En algunos centros el profesorado recibe formación específica y asesoramiento del Departamento de Orientación (PROF6); otros entienden que esta formación debería relacionarse con la metodología (PROF7). En ocasiones necesitan ayuda específica muy concreta "algunos de estos niños sufren acoso, o no se relacionan con los demás" (PROF7), "llegan a ser alumnos con una gran personalidad" (PROF7).

En algunos centros la formación específica se lleva a cabo en Primaria "llevamos 6 años formándonos con el Centro Andaluz de Diferenciación e Intervención en Superdotados” (PROF9); otros a través de iniciativas propias del profesorado (PROF10); otros afirman que "no han recibido formación ni hay gran oferta en el mercado" (PROF8). Se ha avanzado en detección temprana con protocolos al final de la Etapa de Infantil/Primaria y de Primaria/Secundaria, aunque el profesorado necesita formación en habilidades sociales (PROF14) para orientar la intervención educativa. La formación del profesorado (FOR/PROF): debería estar basada en los siguientes elementos teóricos (Cuadro 6).

Cuadro 6

Análisis de entrevistas alumnado: Dimensiones y sub-dimensiones

Metodologías activas y participativas: diseño de respuestas creativas, "no simplemente ampliar fichas y trabajos” (PROF2), y diseño de actividades (PROF 6)

Metodologías innovadoras y motivadoras: "conocer las características de este colectivo, en recursos tecnológicos (apps, robótica, arquitectura...) que a los niños pudieran motivarles” (PROF8)

Planificación de aula: "planificando en función de sus necesidades y de sus capacidades” (PROF3), "formación relacionada con metodología, materiales y evaluación” (PROF12)

Conexión teoría y práctica: el profesorado conoce la teoría "pero al llegar a las clases y al momento de atender a estos alumnos, se sienten totalmente perdidos” (PROF3).

Actitudes ante la enseñanza: para trabajar se deberían contemplar "aspectos actitudinales, saber ponerse en la piel de los alumnos, interesarse realmente por cada uno, adoptando una actitud de preocupación por el otro" (PROF5)

Diseño del currículum abierto, flexible y participativo: “ampliarles los contenidos, adaptar el currículum del alumnado" (PCA10), se trata de "cómo compaginar la tarea con el resto del grupo-clase para que sea efectivo para todos” (PROF11)

Desarrollo de habilidades sociales y aspectos emocionales: conocer las características personales de este alumnado (PROF2) y desarrollar habilidades sociales y afectivas (PROF13)

Nota. Elaboración propia. 


\subsubsection{Red natural de apoyo en el aula (RED)}

Ayuda entre iguales (AYU/IGU): es importante establecer una red natural de apoyo entre compañeros en condiciones adecuadas (PROF1): "son buenos candidatos para llevar a cabo la tutoría entre iguales con alumnos que presentan N.E.A.E” (PROF2). Para algunos centros es una medida de atención a la diversidad la figura del alumno-tutor (PROF3, PROF4) en clase ya que pudiese ser "más significativo y cercano para ellos y considerado por sus compañeros" (PROF5), beneficiando al alumnado-tutor y ayudando a sentirse útiles con otros compañeros (PROF11). Es una estrategia muy positiva (PROF12), que utiliza "un lenguaje de niños, ya que entre ellos se entienden mucho mejor" (PROF14) en el aula ordinaria aumentando el "sentido de pertenencia".

Inclusión en el aula (INC/AU): la inclusión es adecuada, los compañeros/as les tienen mucho respeto y les consideran casi líderes (PROF1): "pueden liderar grupos debido a su capacidad de convicción y persuasión" (PROF12); "están incluidos sin presentar dificultades, integrados totalmente" (PROF3); "son un valor educativo que enriquece a la clase, entendiendo que hay compañeros con capacidades más altas" (PROF7, PROF9, PROF10). En ocasiones son considerados "raritos", "empollones" y "cerebritos" de la clase (PROF4, PROF11), un poco aislados por su manera de ser o relacionarse (PROF5) o conflictivos "por su falta de habilidades sociales y emocionales estando inmersos en numerosos conflictos entre compañeros" (PROF14).

Trabajo colaborativo (TRA/COL): son bien aceptados, aunque hay que trabajar aspectos sociales (PROF2). El profesorado considera que "tienen que estar completamente integrados con sus compañeros y tienen que hacer actividades con ellos" (PROF7) así, el trabajo colaborativo es una ayuda y se apoya tanto a alumnos que les cuesta como alumnos con AACC (PROF10). En los grupos cooperativos (PROF9) comparten actividades y realizan trabajos (PROF14). Algunos centros desarrollan Proyectos para mejorar su atención educativa y ofrecerles una respuesta adecuada, aunque si tienen que salir del aula no estaríamos incluyendo, "este año hemos cambiado la metodología intentando llevar metodologías innovadoras a clase con diferentes niveles de profundización" (PROF8).

Apoyo entre compañeros/as (APY/IGU): con la tutoría entre iguales se pretende motivación y ayuda a sus compañeros (PROF1), pudiendo ser bidireccional y beneficiándose tanto el estudiante tutor/a como el tutorado (PROF10) siendo un instrumento válido para enriquecerse entre ellos (PROF7).

\subsubsection{Relaciones familia-escuela (FAM/ESC)}

Contexto familiar (CON/FAM): el contexto familiar del alumnado se conoce "a través del Informe de Tránsito, información muy básica" (PROF1). Su objetivo es conocer el contexto de cada uno, situación familiar (PROF3) y acercar a la familia en las tutorías (PROF6) para llevar un seguimiento (PROF10), aunque las relaciones familia- escuela pueden estar condicionadas por el tamaño del centro, como ejemplo en centros pequeños existe proximidad al contexto familiar (PROF7). El profesorado lamenta la falta de tiempo para ello ya que "no se dedica todo el tiempo que se debiera a conocer el contexto familiar" (PROF8) entrando en conflicto en ocasiones las relaciones familia-escuela: "las familias ven la escuela como el enemigo que quiere cortarle las alas a su hijo" (PROF5).

Relaciones familia-escuela (RELFE): suelen ser buenas (PROF1), existiendo un protocolo de atención a familias (PCA3, PROF10). En algunos centros la relación familia-escuela es positiva, promueve encuentros e informa (PROF9); las familias muestran interés y preocupación por sus hijos a "nivel emocional y social, que es donde estos alumnos suelen tener dificultades" (PROF11). En ocasiones existen relaciones disruptivas entre centro-familias: "piensan que el colegio debería estar más formado y darles a sus hijos lo que verdaderamente necesitan, los profesores y colegios en general se encogen de hombros diciendo que ellos no pueden hacer nada más" (PROF8) por lo que las relaciones familia-escuela entran en desacuerdo con la atención educativa, "los profesores tienen una gran variedad de alumnos y tienen que atender a todos, el de altas capacidades es listo y puede tirar para adelante, pero el profesor se tiene que dedicar a los que más le necesitan" (PROF8). Las familias discrepan respecto a la intervención educativa, realzan la capacidad de aprendizaje de sus hijos/as y piden atención diferenciada del resto de compañeros, otros no quieren que sus hijos tengan etiquetas para evitar diferencias con los demás (PROF14).

\subsection{Análisis de las entrevistas al alumnado}

El código referido a la experiencia del alumnado con AACC en su vida académica (EXE), indica que las experiencias han sido positivas, están integrados y valorados: "ha sido una experiencia buena, me he sentido feliz y 
cómodo en todos los centros en los que he estado" (ALUM1). En ocasiones indican problemas durante la ESO propios de la adolescencia y no originados por AACC. El segundo código hace referencia a la experiencia del alumnado de AACC con docentes (EXD), asegurando no haber tenido ningún problema. Un estudiante afirma que en su caso los docentes se han mostrado reacios, dudaban de los resultados del diagnóstico pensando que sus AACC eran por estimulación de la familia, "a muchos maestros les chocaba, llegaban a preguntarle a mi madre si ella me incitaba y me explicaba cosas y me motivaba para que yo aprendiera más” (ALUM2).

Respecto a las pruebas de diagnóstico utilizadas (DIAG), todos han pasado pruebas de diagnóstico con diversos profesionales, psicólogos y miembros del Equipo de Orientación Educativa tras las evidencias observadas y, aunque los docentes las han detectado, las familias han sido importantes: "Mi profesora habló con mis padres que sería conveniente que me llevaran al psicólogo y me hicieran las pruebas y estuve un tiempo haciéndome pruebas y me subieron de curso" (ALUM5). Respecto a la comunicación del diagnóstico a las familias y alumnado (COM) para realizar las pruebas de diagnóstico, han contado con su acuerdo: "Mi madre fue al colegio y entonces mi profesora le comentó que no tenía ya más actividades que ponerme porque yo las entendía rápido, las hacía bien” (ALUM3).

Respecto a las reacciones de las familias ante el diagnóstico y primeras propuestas (FAM) afirman que las reacciones son muy diversas, las familias que tenían sospechas decidieron aceptar el diagnóstico y le dieron normalidad, "la actitud fue normal, como si fuera un niño normal, no le dieron gran importancia ni nada" (ALUM3) y familias que insistieron en tomar medidas como flexibilización para evitar fracasos escolares, "regular porque no sabían si me iba a adaptar al cambio" (ALUM4). Dos familias vivieron el proceso con miedo y buscando el bienestar emocional para sus hijos, aspecto que vieron peligrar con la flexibilización. En el código referido a los sentimientos tras conocer el diagnóstico (SENT), estos sentimientos fueron de impacto, alegría y orgullo unido al desconocimiento de lo que significaba tener AACC, optando por darles normalidad.

El código referido a los cambios en la vida académica y social de este alumnado (CAM), aquellos que han vivido más cambios son los que han realizado flexibilización referidos a las amistades; el resto asegura que no tuvieron cambios porque no comentaron su diagnóstico al resto. En el código que analiza las medidas de atención educativa que han recibido (MAT) afirman que el alumnado que ha flexibilizado no ha recibido medidas de atención diferente; los que no han flexibilizado han recibido enriquecimiento curricular fuera del aula ordinaria, en pequeños grupos o individualmente. Todos han aceptado bien los cambios: "Los amigos de la clase de antes a la clase nueva, ya con un año más porque actualmente mis amigos son todos con un año más que yo” (ALUM4).

Respecto a las medidas de flexibilización y respuestas a este alumnado (FLEX), los que aceptaron flexibilización afirman que fue un proceso viviéndolo como algo positivo, los que declinaron la propuesta prefirieron continuar con su ritmo movidos por cuestiones sociales y amistades. En un caso se afirma que hay que "tener cuidado" para implantar esta medida. En la motivación del alumnado en su vida académica (MOT), la mayoría ha experimentado aburrimiento, necesitando un ritmo diferente, "Yo empecé porque volví a casa diciéndole a mi madre que me aburría en clase" (ALUM3). Uno de ellos ha cursado estudios en un centro privado y afirma que es otra historia.

En lo que se refiere a la estimulación recibida (EST), las experiencias son diversas. Algunos no han recibido estimulación externa, han trabajado actividades extraescolares promovidas por sus padres con centros de interés en actividades artísticas, tecnológicas, lingüísticas... "Yo desde que era pequeña siempre he ido al teatro, a conciertos, luego tenía el conservatorio de danza...” (ALUM5). Respecto a si este alumnado ha sido atendido específicamente por profesorado de forma específica (PAT), algunos han contado con atención específica en Educación Primaria; otros afirman que docentes y tutores han ofrecido tareas de ampliación, aunque no trabajando con ningún docente o profesional en particular, "bueno sí, mi tutora dirigía la clase y a mi venía una profesora de apoyo con hueco y la tutora le explicaba lo que tenía que hacer conmigo” (ALUM3).

Respecto al análisis de las relaciones del alumnado de AACC con sus iguales (REL), han sido positivas en general, no han tenido problemas de discriminación en el centro o fuera de él. Algunos se encuentran en un curso superior a su edad y les costó la adaptación, pero se han sentido como "uno más" dentro y fuera del aula y del centro, "han sido totalmente positivas porque los considero mis compañeros y amigos y no he tenido ningún cambio" (ALUM3). En un caso hasta que no accedió a Bachillerato sentía incomprensión, su grupo de iguales no compartía sus intereses, aspecto que cambió cuando accedió al nuevo centro. En cuanto a las opiniones de este alumnado sobre la atención recibida en su vida académica (OAT), estas opiniones son diversas. Algunos afirman que han recibido atención adecuada y no necesitan más, otros les hubiera gustado trabajar mejor. El 
sistema educativo debería mejorar la atención a la diversidad con Proyectos y actividades focalizadas en centros de interés dentro del aula.

Por último, respecto a los consejos que este alumnado proporciona a otros alumnos y al sistema educativo en general (CONS), la mayoría aconseja evitar presumir o alardear de AACC intelectuales y normalizar la situación: "Que no se sienta grande porque al fin y al cabo la grandeza no hace nada" (ALUM3). Los que han flexibilizado aconsejan "arriesgarse" porque contribuye positivamente en su desarrollo y no temer a las nuevas relaciones porque al final te adaptas. Uno de ellos aconseja informar y formar más a las familias ya que el desconocimiento provoca inseguridad para decidir qué medida es positiva para sus hijos.

\section{Discusión y conclusiones}

A continuación, presentamos la discusión y conclusiones de las aportaciones de profesorado y alumnado contrastadas con investigaciones relevantes. La discusión se organiza en torno a una serie de necesidades destacadas por profesorado y estudiantes:

\section{La necesidad de conocer los aspectos emocionales y habilidades sociales del alumnado con AACC}

Los estudios en general afirman que este alumnado podría tener dificultades emocionales, como comprobamos en el trabajo de Kroesbergen y otros (2016), donde se demuestra que el alumnado con AACC dispone de una autoestima y aceptación social algo inferior a la de alumnado con capacidades creativas habituales. Sin embargo, en este estudio los resultados discrepan de esta hipótesis de partida, ya que los propios informantes afirman que el alumnado con resultados académicos excepcionales muestra bienestar psicológico superior, encontrándose incluidos y valorados en el aula ordinaria y en el centro, con experiencias sociales positivas desmitificando el tópico que afirma que son estudiantes aislados, solitarios y rechazados por el grupo de iguales. El alumnado entrevistado indica que existe falta de conocimiento en profundidad de estos estudiantes.

\section{La necesidad de una atención educativa adecuada y específica a través de Programas de enriqueci- miento y flexibilización}

Una atención adecuada es prioritaria para este alumnado, "en la educación hace falta atención a la diversidad tanto por abajo como por arriba" (ALUM5). Kim (2016) ha analizado 26 estudios sobre Programas de Enriquecimiento para estudiantes con AACC demostrando el impacto positivo en sus logros académicos y socioemocionales. De la misma forma Gronostaj y otros (2016) también analizan estos resultados cuando adelantan al alumnado un curso académico. Ningún alumno/a ha experimentado fracaso escolar cuando las medidas de centro, la estimulación externa y atención individualizada fomentan el éxito escolar a través de estas estrategias.

McClarty (2015) demuestra asimismo que este alumnado rinde más cuando se utiliza la aceleración como oportunidades adicionales de estudio avanzado. Las medidas de carácter extraordinario deberían utilizarse cuando no han funcionado las medidas ordinarias (enriquecimiento curricular y/o la flexibilidad horaria). La flexibilización promueve respuestas diversas realizadas de forma procesual, aunque consideran que cambia el ritmo evolutivo del alumnado; otros aseguran que es una medida positiva siempre que se realice de manera escalonada, evitando distorsiones en la vida del alumnado en el ámbito social.

\section{La necesidad de ayudas/apoyos al profesorado en el aula ordinaria}

Se trata de ayudas para responder a estas necesidades dentro del aula ordinaria evitando "trato diferenciado" y propiciando la pertenencia al aula dentro de un marco normalizado e inclusivo de trabajo. Dotar de mayor número de profesores al aula optimizaría la situación de cualquier estudiante que necesite apoyo, "la atención mejoraría si se contara con la presencia de un profesor dentro del aula de forma permanente" (PROF5). Luque-Parra, Hernández-Díaz y Luque-Rojas (2016) han estudiado los aspectos psicoeducativos de la evaluación de este alumnado y concluyen que esta intervención debe estar orientada a describir, explicar y aportar recursos al aprendizaje y el logro de objetivos a través de Programas de Enriquecimiento con la ayuda de un mayor número de profesores en el aula. 


\section{La necesidad de coordinación entre profesorado para unificar criterios de respuesta}

Los estudiantes entrevistados demandan respuestas educativas adecuadas a sus necesidades, lo que exige coordinación entre profesionales para elaborar Planes de Atención a la Diversidad. Es necesario crear grupos de trabajo entre profesores especializados en AACC cuyo objetivo sea ofrecer una respuesta que se ajuste a estas necesidades de forma consensuada. La cultura de la coordinación debe estar integrada en el proceso de enseñanza-aprendizaje y en la planificación del profesorado, no como actuaciones aisladas sino formando parte del trabajo diario. Una de las estrategias de trabajo se basa en crear Redes Educativas entre escuelas para el trabajo en colaboración.

\section{La necesidad de flexibilizar el currículum con estrategias de enseñanza y recursos educativos innova- dores y motivadores en el aula}

Es importante el diseño de actividades "amplias, de libre elección, individuales, de adaptación de materiales y recursos a las necesidades del alumnado" (PROF12). Esta flexibilidad se refiere a la organización del espacio y tiempo, adaptación de metodologías activas y participativas, pero no como un programa individual educativo diferente, si no integradas en el trabajo de aula. El uso de estrategias y recursos específicos se considera imprescindible para afrontar este proceso. El profesorado necesita utilizar "recursos y propuestas creativas desde cada una de las materias para dar respuesta a estas necesidades formativas" (PROF2). Un ejemplo de estrategia innovadora para Fernández del Río y Barreira (2017) son los cortometrajes como herramienta para trabajar en Educación Primaria.

\section{La necesidad de formación del profesorado en AACC}

Esta formación no está regulada en nuestro sistema educativo, se reduce a Programas Formativos puntuales no incluidos en los Planes de Formación previa. Esta demanda de formación especializada debe incluir en los planes formativos a las familias. García-Barrera y de la Flor (2016) han recogido las percepciones del profesorado en relación con la demanda respecto a la atención al alumnado con AACC.

\section{La necesidad de atención a los contextos familiares}

Existen diferencias significativas entre familias en relación con la intervención educativa y sus expectativas. Algunas familias consideran que lo más eficaz es la anticipación de un curso, aunque "hay familias que prefieren que sus hijos queden vinculados a su grupo de iguales" (PROF6). Es necesario destacar que para que la relación familia-escuela sea eficaz es necesario promover el contacto con la familia, pero "siempre y cuando el docente esté preparado" (PROF1). Higueras-Rodríguez y Fernández (2017) reclaman que las familias sean informadas y formadas durante el proceso. La mayoría de familias están satisfechas con las medidas adoptadas, aunque en ocasiones no son informadas adecuadamente y muestran reticencias ante algunas medidas adoptadas.

Entre las conclusiones importantes destacamos que el profesorado es clave para la atención al alumnado con AACC y por ello se necesitan medios personales y formación adecuada para esta atención en los siguientes temas: metodologías activas y participativas, innovadoras y motivadoras, planificación de aula, conexión teoría y práctica, aspectos actitudinales, diseño del currículum abierto, flexible y participativo y habilidades sociales y aspectos emocionales. Se trataría de un Programa de Formación en la atención al alumnado con AACC. Otra cuestión importante es el trabajo colaborativo entre compañeros en clase para propiciar el intercambio entre compañeros, tanto con altas capacidades como alumnado con NEAE. La relación contexto familiar/centro escolar se revela fundamental para el bienestar del alumnado con AACC en los centros escolares.

Este estudio nos revela asimismo que el alumnado con altas capacidades no muestra demasiado descontento en cuanto a la atención recibida en los centros escolares. El alumnado está de acuerdo tanto con las medidas de flexibilización como con los Programas de Enriquecimiento que se han implementado con ellos para la adaptación a sus necesidades. Una de las quejas es quizás la "falta de visibilidad" a la que están sometidos por parte de los centros en la mayoría de las ocasiones y la tardanza en su diagnóstico por parte de la Administración.

En cuanto a las limitaciones de este estudio encontramos la necesidad de ampliar la muestra a las familias para recabar información acerca de esta relación familia/escuela, no obstante, este estudio no pretende extender los resultados y conclusiones a otros contextos. Asimismo, deberíamos ampliar la muestra del alumnado y las preguntas en futuros estudios incluyendo temas de interés como son las estrategias didácticas y los recursos educativos utilizados con este alumnado en su aprendizaje. 


\section{Referencias}

Agudo, N. (2017). Un estudiante con altas capacidades en mi aula, ¿Ahora qué? Revista Nacional e Internacional de Educación Inclusiva, 10(1), 265-277.

Alvarez-Gayou, J. (2005). Cómo hacer investigación cualitativa. Fundamentosy metodología. Paidós.

Cañete-Pulido, M. (2010). La educación en niños y niñas superdotados. Revista Digital de Innovación y Experiencias Educativas, 30, 1-10.

Castellanos, D., Bazán, A., Ferrari, A. M. y Hernández, C. A. (2015). Apoyo familiar en escolares de alta capacidad intelectual de diferentes contextos educativos. Revista de Psicología, 33(2), 299-332.

Delgado, M. y Solano, A. (2009). Estrategias didácticas creativas en entornos virtuales para el aprendizaje. Revista Electrónica Actualidades Investigativas en Educación, 9(2), 1-21.

Fernández del Río, A. B. y Barreira, A. (2017). El cortometraje como herramienta innovadora para el alumnado con altas capacidades en Educación Primaria. Innoeduca. International Journal of Technology and Educational Innovation, 3(1), 2836. https://doi.org/10.24310/innoeduca.2017,v3i1.1962

Fernández, M. C, De Sande, M. J, Martín, A. I. (2006). Medidas de atención a la diversidad para alumnado de altas capacidades. Revista de Educación CEP de Toledo, 1(8), 44-61.

García-Barrera, A. y De la Flor, P. (2016). Percepción del profesorado español sobre el alumnado con altas capacidades. Estudios pedagógicos, 62(2), 129-149.

García-Guardia, M. L., Ayestarán-Crespo, R., López-Gómez, J. y Tovar-Vicente, M. (2019). Educar y formar al alumno talentoso: El afán de logro como competencia curricular. Comunicar, 60(2), 19-28. https://doi.org/10.3916/C60-2019-02

García-Perales, R. y Almeida, L. (2019). Programa de enriquecimiento para alumnado con alta capacidad: Efectos positivos para el currículum. Comunicar, 60(2), 39-48. https://doi.org/10.3916/C60-2019-02

Gronostaj, A., Werner, E., Bochow, E. y Vock, M. (2016). How to learn things at school you don't already know: Experiences of gifted grade-skippers in Germany. Gifted Child Quarterly, 60(1), 31-46.

Guzmán, C. y Saucedo, C. (2015). Experiencias, vivencias y sentidos en torno a la escuela y a los estudios: Abordajes desde las perspectivas de alumnos y estudiantes. Revista Mexicana de Investigación Educativa, 20(67), 1019-1054.

Higueras-Rodríguez, L. (2017). Intervención educativa en el alumnado con altas capacidades. Ensayos Pedagógicos, 12(1), 69-81. https://doi.org/10.15359/rep.12-14

Higueras-Rodríguez, L. y Fernández, J. D. (2017). El papel de la familia en la educación de los niños con altas capacidades intelectuales. International Journal of Educational Reserarch and Innovation, 7, 149-163.

Kim, M. (2016). A meta-analysis of the effects of enrichment programs on gifted students. Gifted Child Quarterly, 60(2), $102-116$.

Kroesbergen, E., Van Hooijdonk, M., Van Viersen, S., Middel-Lalleman, M. y Reijnders, J. (2016). The psychological well-being of early identified gifted children. Gifted Child Quarterly, 6O(1), 16-30. https://doi.org/10.1177/0016986215609113

Luque-Parra, D. J., Hernández-Díaz, R. y Luque-Rojas, M.J. (2016). Aspectos psicoeducativos en la evaluación del alumnado con altas capacidades intelectuales: Análisis de un caso. Summa Psicológica, 13(1), 77-88.

https://doi.org/10.18774/summa-vol13,num1-263

Martínez, S. (2014). La atención educativa a los alumnos de altas capacidades en la etapa de educación primaria desde el marco de la inclusión (Trabajo Fin de Grado). Universidad Internacional de la Rioja, Barcelona.

McClarty, K. (2015). Life in the fast lane: Effects of early grade acceleration on high school and college outcomes. Gifted Child Quarterly, 59(1), 3-13. https://doi.org/10.1177/0016986214559595

Murillo, F. J. y Krichesky, G. (2014). Mejora de la escuela: Medio siglo de lecciones aprendidas. REICE. Revista Iberoamericana sobre Calidad, Eficaciay Cambio en Educación, 13(1), 69-102.

Palomares, A. y García, R. (2016). Innovación y creatividad para favorecer la intervención educativa del alumnado con altas capacidades. Revista de Educación Inclusiva, 9(1), 90-100. 
Ramírez, F. Z. y Soto, B. (2012). Evaluación de la eficacia de tres programas de enriquecimiento de la creatividad. Revista de Investigación y Divulgación en Psicología y Logopedia, 2(2), 35-40.

Seijo, J. C. T., López, C. M., Pedrajas, M. L. y Martínez, C. (2015). Formación del profesorado en aprendizaje cooperativo y alumnos con altas capacidades: Un enfoque inclusivo. Revista Latinoamericana de Educación Inclusiva, 9(2), 91-110.

Tojo, C., Santos, G., Nores, A. I., Cao, L. R. y Quintela, L. R. (2017). Los programas de enriquecimiento: Desarrollo integral de las altas capacidades. International Journal of Developmental and Educational Psychology, 6(1), 27-32. https://doi.org/10.17060/ijodaep.2014.n1.v6.714

Torrego, J. (2011). Alumnado con altas capacidades y aprendizaje cooperativo. Fundación SM.

Torrego, J. C., Monge, C., Pedrajas, M. y Martínez, C. (2015). Formación del profesorado en aprendizaje cooperativo y alumnos con altas capacidades: Un enfoque inclusivo. Revista Latinoamericana de Educación Inclusiva, 9(2), 91-110.

Vallejo, P. y Morata, M. (2015). Intervención psicoeducativa en un caso de altas capacidades. Revista de Psicología Clínica con Niñosy Adolescentes, 2(1), 69-74.

\section{Breve CV de las autoras}

\section{Elena Hernández de la Torre}

Profesora Titular de la Universidad de Sevilla (España), Facultad de Ciencias dela Educación. Profesora del Máster Universitario en Psicopedagogía, miembro del Programa de Doctorado en Educación y miembro del Instituto Interuniversitario de Investigación Educativa. Líneas de investigación: atención a la diversidad, Proyectos de Mejora Educativa en centros escolares, dificultades en el aprendizaje escolar e intervención pedagógica y formación del profesorado. Miembro del Grupo de Investigación, Desarrollo, Evaluación y Asesoramiento IDEA (HUM-423). Email: eht@us.es

ORCID ID: https://orcid.org/0000-0001-6390-1955

\section{Ma José Navarro Montaño}

Profesora Titular de Universidad, profesora del Máster Universitario en Necesidades Educativas Especiales y Atención a la Diversidad en la Escuela y miembro del Programa de Doctorado en Educación de la Facultad de Ciencias de la Educación, de la Universidad de Sevilla (España). Doctora en Filosofía y Ciencias de la Educación. Líneas de investigación: Educación inclusiva, Atención a la diversidad y Necesidades Específicas de Apoyo Educativo. Miembro del Grupo de Investigación Didáctica: Análisis tecnológico y cualitativo de los procesos de enseñanza-aprendizaje (HUM-390). Las investigaciones recientes se enmarcan en Proyectos sobre Redes Educativas de Centros Escolares y Altas Capacidades Intelectuales. Email: maripe@us.es

ORCID ID: http://orcid.org/0000-0003-2462-8348 